\title{
Potential-vorticity inversion and the wave-turbulence jigsaw: some recent clarifications
}

\author{
M. E. McIntyre \\ Centre for Atmospheric Science at the Department of Applied Mathematics and Theoretical Physics, Wilberforce Road, \\ Cambridge CB3 0WA, UK
}

Received: 17 March 2008 - Revised: 18 June 2008 - Accepted: 19 June 2008 - Published: 25 June 2008

\begin{abstract}
Two key ideas stand out as crucial to understanding atmosphere-ocean dynamics, and the dynamics of other planets including the gas giants. The first key idea is the invertibility principle for potential vorticity (PV). Without it, one can hardly give a coherent account of even so important and elementary a process as Rossby-wave propagation, going beyond the simplest textbook cases. Still less can one fully understand nonlinear processes like the selfsharpening or narrowing of jets - the once-mysterious "negative viscosity" phenomenon. The second key idea, also crucial to understanding jets, might be summarized in the phrase "there is no such thing as turbulence without waves", meaning Rossby waves especially. Without this idea one cannot begin to make sense of, for instance, momentum budgets and eddy momentum transports in complex large-scale flows. Like the invertibility principle the idea has long been recognized, or at least adumbrated. However, it is worth articulating explicitly if only because it can be forgotten when, in the usual way, we speak of "turbulence" and "turbulence theory" as if they were autonomous concepts. In many cases of interest, such as the well-studied terrestrial stratosphere, reality is more accurately described as a highly inhomogeneous "wave-turbulence jigsaw puzzle" in which wavelike and turbulent regions fit together and crucially affect each other's evolution. This modifies, for instance, formulae for the Rhines scale interpreted as indicating the comparable importance of wavelike and turbulent dynamics. Also, weakly inhomogeneous turbulence theory is altogether inapplicable. For instance there is no scale separation. Eddy scales are not much smaller than the sizes of the individual turbulent regions in the jigsaw. Here I review some recent progress in clarifying these ideas and their implications.
\end{abstract}

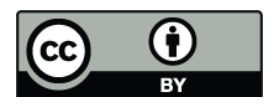

Correspondence to: M. E. McIntyre (www.atm.damtp.cam.ac.uk/mcintyre)

\section{Introduction}

This paper is dedicated to the memory of Rupert Ford, my brilliant ex-student who died tragically in diabetic coma at the Nice EGS Assembly of March 2001. Rupert was approaching the peak of his powers, an international research star whose sudden death was a terrible loss to science. He'll be remembered not only for his warm humanity and for his generosity and engagement with colleagues, not least at EGS Assemblies, but also for his passion to understand things.

Here I mean fully understand, in the deepest scientific sense aimed at and powerfully communicated by the likes of Einstein, Prandtl, Lighthill, Feynman, and my own thesis supervisor Francis Bretherton and his supervisor, the legendary Sir Geoffrey Ingram Taylor. James Gleick's book on Feynman (Gleick, 1992) brilliantly conveys what such understanding involves. I have tried in my own way to say it in my writings on "lucidity principles", as well as in Rupert's obituary (McIntyre, 2001). Understanding in this sense involves seeing things from as many angles as possible and finding insightful thought experiments - playing with the system or parts of it to see what happens, like a child playing with elastic bands or Lego or Meccano - and in particular keeping words, logic, pictures, mechanistic feelings, mathematical symbols and equations tied together into a coherent and selfconsistent whole. ${ }^{1}$

Along with such understanding goes a sense of what's robust. Rupert and I always felt that a theoretician's job is not only to study the special cases described by idealized models, using whatever mathemematical ingenuity it takes, but also

\footnotetext{
${ }^{1}$ In Gleick's book see especially The Aura, around p. 131, and A half-assedly thought-out pictorial semi-vision thing, around p. 245. (I disagree, though, that quantum theory forces us to abandon the usual assumption of a single outside reality, the basis of all science, p. 243. Rather, it forces us to be meticulous in distinguishing between reality on the one hand, and our models of reality on the other, thus clarifying what we know about reality and, indeed, what we can know about reality - websearch "coherent account" "thinking probabilistically”.)
} 
to try to discern what's robust about a given model - that is, which of its qualitative features are likely to be shared with more realistic and complicated models and with reality itself. An elementary illustration is the Rossby-wave propagation mechanism, central to practically everything about the dynamics of the atmosphere-ocean system.

It is one thing to derive the Rossby-wave dispersion relation in idealized cases where the mathematics is easy, in the manner of most textbooks. It is quite another to understand why the propagation mechanism works equally well in the more realistic and complicated cases for which no simple mathematical solutions are available.

Common to all these cases, idealized and realistic, is the peculiar quasi-elasticity of potential-vorticity (PV) contours and, for instance, the role of that quasi-elasticity, along with shear, in the dynamics of what are now called eddy-transport barriers - helping to explain the robustness of strong jets and vortices like those on Jupiter and the other gas giants, and the tendency for the eddy transport of material tracers to be inhibited across prograde jet cores or vortex edges even when the Eulerian velocity field involves aperiodic, large-amplitude disturbances, going beyond the purely kinematic, Kolmogorov-Arnol'd-Moser theory of barrier formation (e.g., Haynes et al., 2007; Rypina et al., 2007; BeronVera et al., 2008).

A full understanding of such phenomena can scarcely be arrived at without the conscious or unconscious use of the PV "invertibility principle" or something equivalent (e.g., Hoskins et al., $1985 \&$ refs). It is an essential component of the conceptual "Lego set" one needs.

PV invertibility says that, to sufficient approximation, the flow is balanced such that all the other fields can be derived diagnostically from the PV field, that is, derived at each instant of time independently of what goes on at earlier or later times. In other words, there is such a thing as a diagnostic "PV inversion operator". This is a nonlocal, timeindependent function whose input is the PV field at a given instant, and whose output consists of the other fields at that instant, such as the velocity and buoyancy fields.

In the case of the Rossby-wave mechanism, for instance, PV invertibility gives us a way of saying that the sideways undulation of PV contours instantly implies a velocity field across the contours that's a quarter wavelength out of phase with the undulatory displacement field. This in turn implies, in a perfectly definite way, the familiar one-way phase propagation and all its far-reaching consequences stemming from the single time derivative in the evolution equation for PV. It is crucial that the velocity field is linked, diagnostically, to the displacement field and the implied pattern of PV anomalies, in such a way as to keep the displacement and velocity fields phase-locked a quarter-wavelength apart as the undulations propagate.

More generally, as stated in Ford et al. (2000), PV invertibility and the associated ideas of balanced flow and slow quasimanifold allow one "to treat the central difficulty of fluid dynamics, the advective nonlinearity, with maximum possible simplicity by representing it solely in terms of the advection of potential vorticity and near-surface potential temperature." Moreover the ideas "make explicit and keep conceptually separate" the "prognostic versus diagnostic, advective versus nonadvective, and local versus nonlocal" aspects of the dynamics.

The nonlocal aspects are made explicit through the idea of PV inversion. They are made explicit in such a way as to evade the limitations and inaccuracies of highly idealized models such as quasigeostrophic theory. We can therefore use the qualitative insights from such idealized models in the knowledge that those insights will be robust and will carry over to more sophisticated models in which the PV is evaluated from its most accurate (Rossby or Ertel) formula, and inverted and advected as accurately as possible.

None of this seems to be brought out with complete lucidity in the textbooks I've seen. In fairness, however, there were until recently some unresolved technical difficulties with the most accurate known PV inversion operators.

Those difficulties, which I believe were first fully spelt out in Mohebalhojeh (2002), were finally overcome - as I first reported at EGU 2006 - with the discovery of a family of "hyperbalance equations" (Mohebalhojeh and McIntyre, 2007a, $\mathrm{b}$, hereafter MM07a, b). The corresponding PV inversion operators are free of the difficulties in question.

Section 5 below presents and briefly discusses the simplest member of the family. Before that, Sects. 2-4 recall a few points about the nonlinear jet problem and the "Rhines effect", supplementing the more extensive review in Dritschel and McIntyre (2008, hereafter DM08, in the J. Atmos. Sci. Special Collection on Jets and Annular Structures in Geophysical Fluids). In conclusion, Sect. 6 suggests a need for forced geophysical turbulence experiments to consider momentum or impulse injection, as well as energy injection. There is a concomitant need to consider more explicitly the radiation-stress field due to Rossby waves and other wave types. $^{2}$ Examples where that need is especially conspicuous include the giant planets' equatorial prograde jets.

\footnotetext{
${ }^{2}$ The term "radiation stress" is used here as in DM08 to mean wave-induced momentum transport regardless of wave type, as is standard in the language of physics. In atmosphere-ocean dynamics the most important wave types, for this purpose, are Rossby and gravity waves and their equatorial hybrids, not acoustic or electromagnetic waves. The importance of radiation stresses illustrates one of the grand themes of physics, the dynamical organization of fluctuations with systematic mean effects. Any wave propagation mechanism will tend to organize the fluctuating fields, no matter how chaotic they may seem, in the sense of inducing systematic correlations among the components of those fields. The correlations are shaped by the waves' polarization relations and usually give rise to systematic, long-range momentum transports. For completness it is useful to include under the "radiation stress" heading the stresses due to wave diffraction; indeed, for instance with planetary-scale Rossby waves, the distinction can sometimes be somewhat blurred.
} 


\section{The sharp-jet attractor}

The word "turbulence" tends to make us forget how simple, robust, and easy to understand is the once-mysterious antifrictional self-sharpening of prograde jets - what used to be called "negative viscosity". Such self-sharpening is a large part of why narrow jets or jetstreams are so persistent, and so ubiquitous, in the atmosphere and oceans. The selfsharpening phenomenon is incomprehensible in terms of the old pure-turbulence or eddy-viscosity (momentum-mixing) paradigms. One reason is that those paradigms ignore the crucial role of Rossby-wave dynamics, first recognized by R. E. Dickinson (1969) and E. T. Eady (unpublished).

On a qualitative level, the self-sharpening phenomenon is almost trivial to understand if one uses PV invertibility and the idea that PV mixing - as distinct from momentum mixing - is kinematically favoured on one or both flanks of the jet but inhibited in the jet core, where not only flow speeds but also PV gradients and Rossby-wave elasticity are strongest. DM08 give a historical review that goes back to the pioneering work of H. Jeffreys, V. P. Starr, N. A. Phillips, O. M. Phillips, and Dickinson and Eady, and points to today's wealth of observational and modelling evidence for the success of such understanding, helping us to make sense of observed flows in the terrestrial and Jovian atmospheres and in the terrestrial oceans.

The evidence is especially precise, plentiful, and well studied in the case of the terrestrial stratosphere because of its bearing on the stratospheric ozone problem. To see a movie of the real stratosphere doing its jet-sharpening on a grand scale, from the work of Riese et al. (2002), websearch "gyroscopic pump in action". Note the near-perfect mixing over a vast area on the equatorward flank of the polar-night jet. Beyond the DM08 review, this qualitative picture of jet selfsharpening through the interplay of waves and turbulence has been put into a more quantitative form, retaining its simplicity and comprehensibility, in the beautiful recent work of Esler (2008).

Central to all this is the robustness and wide applicability of a remarkably simple jet model, the well known shallowwater "contour-dynamics jet" or "equivalent barotropic thin jet" that results from inverting a simple PV discontinuity or idealized front, i.e. a jet with perfectly-mixed PV on both flanks and a delta-function PV gradient in its core. In its simplest, quasigeostrophic $f$-plane description, the inversion gives the sharply-peaked velocity profile

$\bar{u}(y)=U \exp \left(-L_{\mathrm{D}}{ }^{-1}|y|\right) \quad$ with $\quad U=\frac{1}{2} L_{\mathrm{D}} \Delta q$

where $L_{\mathrm{D}}$ is the Rossby deformation length, $\Delta q$ the jump in quasigeostrophic PV, and $y$ the transverse distance relative to the front or jet core. More accurate inversions all give a qualitatively similar structure. Finite $L_{\mathrm{D}}$ is a natural assumption for planetary atmospheres lacking rigid lids, and oceans in which the significant eddy dynamics is associated with internal or baroclinic $L_{\mathrm{D}}$ values.
Why should (1) be a robust model of real jets? The reason is that the Rossby waves propagating on the concentrated PV gradient at the jet core always have phase speeds $c$ in the range $(0, U)$. Therefore there are always critical lines in the jet flanks. It is a standard class exercise to verify this. The linearized quasigeostrophic $\mathrm{PV}$ equation is

$q_{t}^{\prime}+\bar{u} q_{x}^{\prime}+\bar{q}_{y} \psi_{x}^{\prime}=0$

$\left(\psi^{\prime}=\mathcal{L}^{-1} q^{\prime} ; \quad \mathcal{L}:=\partial_{x}^{2}+\partial_{y}^{2}-L_{\mathrm{D}}{ }^{-2} ; \quad \bar{q}_{y}=-\mathcal{L} \bar{u}\right)$

with $x$ along the jet, the overbars denoting averages along the jet. For wavenumber $k$ along the jet and disturbance streamfunction $\psi^{\prime} \propto \hat{\psi}(y) \exp \{i k(x-c t)\}$ one finds

$$
c=U\left\{1-\left(1+L_{\mathrm{D}}^{2} k^{2}\right)^{-1 / 2}\right\} \approx \frac{1}{2} U L_{\mathrm{D}}^{2} k^{2} \text { when } k \ll L_{\mathrm{D}}{ }^{-1}
$$

along with $\hat{\psi}(y)=\exp \left\{-\left(L_{\mathrm{D}}^{-2}+k^{2}\right)^{1 / 2}|y|\right\}$, describing the undulations of the jet. A similar long-wave asymptotics in which $c \rightarrow 0$ through positive values, like $k^{2}$ as $k \rightarrow 0$, with $\hat{\psi}(y) \propto \bar{u}(y)+O\left(k^{2}\right)$, holds for arbitrary isolated-jet profiles with $\bar{u}(y) \rightarrow 0$ as $|y| \rightarrow \infty$ (Simmons, 1974). Moreover, this qualitative asymptotic behaviour is sufficiently robust to survive generalization to large-amplitude undulations, and to dynamical descriptions more accurate than quasigeostrophic (e.g., Nycander et al., 1993 \& refs).

The presence of critical lines means that, whenever the jet is disturbed, its undulations - more precisely, the undulations of the material and PV contours marking the jet core - will automatically be accompanied by irreversible mixing or rearrangement of material particles in the flanks of the jet to either side. The mixing arises from the existence of hyperbolic points in the co-moving Kelvin-cat's-eye flow patterns in the jet flanks. It exemplifies the most important kind of Rossby-wave breaking (e.g., McIntyre and Palmer, 1985; Polvani and Plumb, 1992), whose dynamical significance for the wave-turbulence interplay was originally illuminated, as is well known, by the Stewartson-Warn-Warn (SWW) theory of nonlinear Rossby-wave critical layers or idealized surf zones (Stewartson, 1978; Warn and Warn, 1978; Killworth and McIntyre, 1985; Haynes, 1989).

When amplitudes increase to realistic values, with sideways slopes of order unity or more, the surf zones in the jet flanks expand to widths of order $L_{\mathrm{D}}$ or more, as is well illustrated in the gyroscopic-pumping movie and in a vast repertoire of numerical experiments (e.g., Held and Phillips, 1987; Polvani and Plumb, 1992; Esler, 2008). One consequence is that there is no turbulent scale separation. Surf-zone eddy or nominal cat's-eye scales transverse to the jet are no smaller than the jet scale itself, $L_{\mathrm{D}}$.

So the jet, when disturbed in almost any natural way, has a strong tendency to mix the PV in its flanks and thus to keep itself sharp, even if not quite as sharp as in the idealization (1). If for any reason the PV gradient in the jet core were to be smeared out over widths of order $L_{\mathrm{D}}$ or more, so that 
the jet became significantly broader, as dictated by PV inversion, then mixing in the jet flanks would reverse that process by expelling PV contours from the flanks and pushing them back into a narrowing core region. Inversion then says that the velocity profile must go back toward (1). The jet self-sharpening process is fundamentally similar to the process called vortex-edge erosion or stripping (e.g., Juckes and McIntyre, 1987; Legras et al., 2001 \& refs).

These considerations go far toward explaining the tendency toward strong spatial inhomogeneity observed in turbulent flows in the real atmosphere and oceans and in the outer layers of the gas giants such as Jupiter. Once jets are established, they tend to maintain themselves as just described. And in a thought experiment in which one starts with no jets, i.e. with statistical homogeneity in space, the positive feedback from the "PV Phillips effect" (O. M. Phillips, 1972; DM08; Haynes et al., 2007) predisposes the flow to develop spatial inhomogeneities and therefore incipient jets. Sideways PV mixing in a given region or zone weakens its Rossby quasi-elasticity and thereby - radiation stresses permitting (Sect. 6 below) - facilitates further mixing there. This is in partial analogy to the weakening of gravity-wave elasticity by vertical mixing in a given layer within a density-stratified fluid, the original "Phillips effect" leading to spontaneous "density layering". However, in the Rossbywave case the shear effects from the jets reinforce the positive feedback.

In dynamical-systems terms we may say heuristically that the system tends to be attracted toward a "PV staircase", for which PV inversion dictates sharp prograde and broad retrograde jets. Evidence for such a tendency includes, for instance, the above-cited numerical experiments on sharpening and stripping, and the work of Marcus (1993), Cho and Polvani (1996), Peltier and Stuhne (2002), Humphreys and Marcus (2007), Scott and Polvani (2007), DM08, and for large $L_{\mathrm{D}}$ - rigid or nearly-rigid lids - the laboratory experiments of Sommeria et al. $(1989,1991)$ and Read et al. (2007) and the numerical experiments of Marcus and Lee (1998), Danilov and Gurarie (2004), and Danilov and Gryanik (2004).

\section{The Rhines effect revisited}

The naturally strong spatial inhomogeneity of such PVstaircase flows, and in particular the lack of scale separation, have implications for turbulence theories (e.g., Baumert et al., 2005). One is led to question the privileging of Fourier analysis and power spectra as the chief way of describing these flows - especially power spectra, which throw away all phase information and thus hide coherent structures such as sharp jets (Armi and Flament, 1985). In particular, given that Rossby waves are important, Fourier analysis is a natural tool only when the Rossby waves behave as superpositions of plane waves, i.e., when they behave as if propagating on a uniform PV gradient. If, however, the PV contours are really bunched up into the prograde jet cores, then the prograde jets become waveguides and the Rossby-wave dispersion relation is much better approximated by (3). This differs from the plane-wave dispersion relation and implies different scalings and power law dependences. The differences come about precisely because of the spatial inhomogeneity of the PV gradient.

The term "Rhines effect" is sometimes used to mean that Rossby-wave dynamics and turbulent dynamics are simultaneously important at some length scale $L_{\mathrm{Rh}}$, the Rhines scale. In PV-staircase flows, dominated as they are by the Rossby elasticity of sharp jets like (1), the standard, plane-wavebased estimates of $L_{\mathrm{Rh}}$ are irrelevant. The more accurate dispersion relation (3) is, by contrast, closely relevant. And precisely because (3) implies Rossby-wave phase speeds $c$ in the same range as the jet's flow speeds $\bar{u}(y)$, and because this relationship is robust even for large-amplitude undulations of the jet, the wave dynamics and turbulent mixing are indeed simultaneously important and, moreover, closely interdependent in the manner already explained. One might say that waves and turbulence are in a tight symbiosis, and that it is therefore a case of the Rhines effect.

This is no surprise in view of the fundamentally similar symbiosis in the SWW critical-layer problem. In the sharpjet problem, in which the scale $L_{\mathrm{D}}$ characterizes both the prograde jet width and the wave-turbulence symbiosis, $L_{\mathrm{Rh}}$ in the above sense must clearly be equated with $L_{\mathrm{D}}$.

In power-spectral terms one can imagine starting with a statistically homogeneous turbulent flow exhibiting the standard upscale energy cascade. In cases like those considered here, where the system is attracted toward a PV staircase, one can reasonably say that the upscale energy cascade has thereby been arrested or at least slowed down. And if in the usual way $L_{\mathrm{Rh}}$ is defined as the lengthscale associated with the dynamics of such arrest or slowdown, then one would again have to define it simply as $L_{\mathrm{D}}$. Arguably, however, it might be more useful to define $L_{\mathrm{Rh}}=L_{\mathrm{Rh}}(U):=(U / \beta)^{1 / 2}$ where $U$ is some chosen velocity scale, and where $\beta$ denotes the background PV gradient, the $\bar{q}_{y}$ of the initial, statistically homogeneous state. With the choice (1) for $U$ we then have yet another scale, $\max \left(L_{\mathrm{Rh}}, L_{\mathrm{Rh}}{ }^{2} / L_{\mathrm{D}}\right)$, for the jet spacing or PV staircase step size or retrograde jet width (DM08, Sect. 6) - even though this, too, is sometimes called the Rhines scale.

\section{Straight and meandering jets}

Scott and Polvani (2007) have presented an interesting forced-dissipative shallow-water model study of Jupiter's jets, in which it is clear from PV maps (their Fig. 9b, c) that the jets meander strongly in high latitudes where $L_{\mathrm{D}}$ values are smallest. The real planet's high-latitude jets meander much less, so may correspond to a model either with larger 
$L_{\mathrm{D}}$ or, perhaps more likely, with weaker forcing and weaker dissipation. In low latitudes the jets have less room to meander, having a spacing not much greater than the local $L_{\mathrm{D}}$ by the usual estimates, and indeed are strikingly straight in the model just as they are on the real planet.

The high-latitude meandering in the model resembles that of the principal jets in the terrestrial oceans such as the Gulf Stream, Agulhas and Kuroshio currents (e.g., Niiler et al., 2003), and of the jets in the Antarctic Circumpolar Current (e.g., Hughes, 1996), for all of which the effective $L_{\mathrm{D}}$ values are small enough for the jets to behave as if isolated, thus having plenty of room to meander.

Having plenty of room is not enough by itself, of course. Also relevant are topographic forcing and the long-wave asymptotics of Eq. (3) and its generalizations. The corresponding group velocity along the jet is asymptotically $\frac{3}{2} U L_{\mathrm{D}}^{2} k^{2}$, tending to zero with respect to the jet surroundings. So an isolated jet viewed on large scales $\gg L_{\mathrm{D}}$ has a flaccidity related to this low group velocity and to the short range $O\left(L_{\mathrm{D}}\right)$ of the $\mathrm{PV}$ inversion operator, $\mathcal{L}^{-1}$ in Eq. (2b). The jet is almost like an elastic thread under zero tension, or a tube made of some material that's hard to cut but easy to bend and stretch. It is a coherent structure that hangs together strongly while being easy to push sideways, to form a large-amplitude undulation or river-like meander that has little influence either upstream or downstream.

Indeed, so large can such meanders become that their sideways slopes can go through infinite and then negative values, forming loops that close on themselves and reconnect, billabong-like, to spawn "Gulf Stream rings", that is, vortices with cores significantly larger than $L_{\mathrm{D}}$. Similarly large vortices are much in evidence at high latitudes in Scott and Polvani's (2007) Fig. 9c, for which $L_{\mathrm{D}}$ at the pole is $1 \%$ of Jupiter's radius, about 3 times smaller than values thought to be realistic. The more realistic Fig. $9 \mathrm{~b}$ shows meanders but fewer vortices.

The nonlinear solutions shown in Nycander et al. (1993), for the idealized sharp jet, have a very similar looping behaviour; and they show that the smallness of the group velocity for small wavenumber $k \ll L_{\mathrm{D}}{ }^{-1}$, hence the flaccidity of isolated jets viewed on large scales, is another property that survives generalization to large-amplitude meanders. For larger values of $k \sim L_{\mathrm{D}}{ }^{-1}$, (3) implies that the jets are, by contrast, relatively stiff, helping to account for the extreme straightness of Jupiter's low-latitude prograde jets.

\section{The hyperbalance equations}

So how far can we push the concepts of balance and PV invertibility? And what does it take to do accurate PV inversion in a completely consistent way? As the foregoing illustrates, along with countless other examples, it is difficult to imagine a more fundamental pair of questions about atmosphere-ocean dynamics (e.g., Hoskins et al., 1985). The simplest context in which the question is nontrivial is that of the shallow-water equations. I still remember my astonishment in the 1980s at seeing, in that context, just how accurate inversion can be - how nearly complete is the dynamical information contained in the PV field - in parameter conditions far outside those in which balance and invertibility are justifiable through formal asymptotics. For instance, local Froude and Rossby numbers could be pushed to somewhere near unity. Thus, flow speeds could be of the same order, numerically, as local gravity-wave speeds. This discovery emerged from the thesis work of Warwick Norton, a research student working with me at the time (Norton, 1988; see also McIntyre and Norton, 1990, 2000).

There followed a rather slow and tortuous history of efforts to make sense of the discovery and to better understand the properties of accurate PV inversion and balanced evolution, with partial success. The accuracy has something to do with the relative smallness of $L_{\mathrm{D}}$ under the more extreme parameter conditions, making jets thin and flaccid and PV inversion a short-range operation. The flaccidity seems to persist as Froude and Rossby numbers increase toward unity. Since it's relative to the jet surroundings that the long-wave group velocity is low, the Galilean-invariance conundrum discussed in Sect. 7 of McIntyre and Norton (2000) also becomes easier to understand. The most accurate balance conditions are pivoted around $\partial / \partial t$, as in Eqs.(10) below, rather than around the material time derivative. And $\partial / \partial t$ measures the unsteadiness of the meander shapes, for which the timescales tend to be longer than for the river-like flow through the meanders.

Another line of attack, in which Rupert Ford led the way, was to try to understand a related phenomenon, the weakness of spontaneous imbalance, the spontaneous-adjustment emission of inertia-gravity waves by unsteady vortical motions. Again, thin-jet flaccidity helps to explain why such wave emission is even weaker than hinted at by the classical Lighthill-type theories. Rupert's last publication in this area was posthumous (Ford et al., 2002). But I don't think anyone has fully penetrated any of these problems, as yet, despite efforts going back to the 1996 Isaac Newton Institute Programme on the Mathematics of Atmosphere and Ocean Dynamics and more recently at the 2006 Seattle workshop on Spontaneous Imbalance, now the topic of another J. Atmos. Sci. Special Collection to which I contributed.

There has, however, been one significant recent advance in our understanding of accurate PV inversion operators, which I first reported at EGU 2006 and will now sketch briefly. In the course of all these efforts another colleague, Ali Mohebalhojeh, noticed that, despite their prodigious success, all the accurate PV operators then known have an inconsistency built into them, underlying the technical difficulties flagged in Sect. 1 (Mohebalhojeh, 2002). For more about the tortuous history, the interested reader can websearch on the phrases "discovered to my embarrassment" and "non-Hamiltonian velocity splitting". To cut a long story short, the upshot was 
the discovery of a new class of highly accurate PV inversion operators and associated balanced models that are free of all such difficulties (MM07a,b). "Velocity splitting" refers to the fact that, implicitly, all the older accurate balanced models have two velocity fields, one to advect mass and the other to advect and evaluate the PV. The difference was tiny, well hidden within numerical truncation errors, but - unlike the different kind of velocity splitting found in Hamiltonian balanced models (McIntyre and Roulstone, 2002) - it precluded an exactly-consistent PV inversion operation.

The new balanced models were given the generic name "hyperbalance equations" because they can be viewed as natural generalizations, to arbitrary formal accuracy, of the Bolin-Charney "balance equations" which, as is well known, are accurate to only two orders in the Froude and Rossby numbers. Even in their simplest versions, the hyperbalance equations are highly implicit. Moreover, they are integrodifferential equations involving variational or functional differentiation. That could be why they were not discovered long ago. MM07a defines them for continuously stratified as well as for shallow-water systems.

The formally simplest member of the class, which applies to $f$-plane shallow water with no bottom topography, may be stated as follows. The single prognostic equation is

$$
\frac{\partial Q}{\partial t}=-\mathbf{u} \cdot \nabla Q
$$

material invariance of $\mathrm{PV}$, where $Q$ is the exact $\mathrm{PV}$. For the shallow-water system the velocity field is $\mathbf{u}=(u, v)$, a function of horizontal position $\mathbf{x}=(x, y)$ as well as of time $t$, and $\nabla:=\left(\partial_{x}, \partial_{y}\right)$, while the exact PV is defined by

$$
Q:=\frac{f+\zeta}{H+h}
$$

(Rossby, 1936). Here $f$ is the constant Coriolis parameter, $\zeta$ the relative vorticity, $H$ the area-mean layer depth and $h$ the variable free-surface departure from $H$, such that $H+h>$ 0 . It will be convenient to define $L_{\mathrm{D}}$ as exactly $(g H)^{1 / 2} / f$ where $g$ is the gravitational acceleration, even though $h$ need not be small in any sense. Denoting the divergence by $\delta$, we use a Helmholtz decomposition

$$
\mathbf{u}=\mathbf{u}_{\zeta}+\mathbf{u}_{\delta} \text { where } \mathbf{u}_{\zeta}:=\hat{\mathbf{z}} \times \nabla \nabla^{-2} \zeta \text { and } \mathbf{u}_{\delta}:=\nabla \nabla^{-2} \delta
$$

with suitable boundary conditions; here $\hat{\mathbf{z}} \times \nabla:=\left(-\partial_{y}, \partial_{x}\right)$.

In order that the single velocity field $\mathbf{u}$ shall exactly advect mass, as well as exactly advecting and evaluating the PV, the divergence field $\delta$ must satisfy the implicit elliptic equation

$$
\mathcal{L} \delta=\frac{1}{L_{\mathrm{D}}^{2}} \nabla \cdot\left(\frac{\mathbf{u} \zeta}{f}\right)-\nabla^{2} \nabla \cdot\left(\frac{\mathbf{u} h}{H}\right)+\frac{\partial \mathcal{R}}{\partial Q} \odot(\mathbf{u} \cdot \nabla Q)
$$

where $\mathcal{L}$ denotes $\nabla^{2}-L_{\mathrm{D}}{ }^{-2}$, the same modified Helmholtz operator as in Eq. (2b). For a detailed derivation and motivation the reader may consult MM07a. In the last term, $\mathcal{R}$ is a nonlocal function of the $Q$ field to be defined shortly, and $\partial$ denotes variational differentiation with respect to that nonlocal dependence. The last term is crucial to eliminating the inconsistency found by Mohebalhojeh. This was proved in MM07a. See Sect. 3 therein, and the end of Sect. 4 therein. The symbol $\odot$ denotes the unweighted inner product over the physical domain, defined such that the small variation $\partial \mathcal{R}(\mathbf{x})$ in $\mathcal{R}$ induced by an arbitrary small variation $\partial Q(\mathbf{x})$ is given by

$$
\partial \mathcal{R}(\mathbf{x})=\frac{\partial \mathcal{R}}{\partial Q} \odot \partial Q:=\iint \frac{\partial \mathcal{R}\{\mathbf{x} ; Q(\cdot)\}}{\partial Q\left(\mathbf{x}^{\prime}\right)} \partial Q\left(\mathbf{x}^{\prime}\right) d x^{\prime} d y^{\prime}
$$

where the integral is taken over the physical domain.

In order to define $\mathcal{R}$ itself we need to introduce a set of auxiliary variables, to be denoted by $\delta_{\mathrm{a}}^{(n)}, \zeta_{\mathrm{a}}^{(n)}$, and $h_{\mathrm{a}}^{(n)}$. They can be regarded as diagnostic estimates of the time derivatives $\partial^{n} \delta / \partial t^{n}, \partial^{n} \zeta / \partial t^{n}$, and $\partial^{n} h / \partial t^{n}$. Being purely diagnostic quantities, they are not to be confused with true time derivatives. With the sole exception of (4) the equations are strictly diagnostic. In order to have a balanced model in the standard sense, it is crucial that (4) be the only prognostic equation in the system.

Allowing at least some of the diagnostic estimates to differ from the true time derivatives - even if only by tiny amounts - is also crucial both to attaining high accuracy and to eliminating the inconsistency found by Mohebalhojeh. In particular $\delta_{\mathrm{a}}^{(1)}$ means a diagnostic estimate of $\partial \delta / \partial t$, and $\delta_{\mathrm{a}}^{(0)} \mathrm{a}$ diagnostic estimate of $\delta$ itself; and it is crucial that the corresponding values be allowed to differ slightly. ${ }^{3}$ By contrast, in this simplest version of the hyperbalance equations we can, and do, consistently take $\zeta_{\mathrm{a}}^{(0)}=\zeta$ and $h_{\mathrm{a}}^{(0)}=h$.

We may now define $\mathcal{R}$ by

$\mathcal{R}=\frac{-1}{f^{2} L_{\mathrm{D}}^{2}}\left\{\delta_{\mathrm{a}}^{(1)}+\nabla \cdot\left(\mathbf{u}_{\mathrm{a}}^{(0)} \cdot \nabla \mathbf{u}_{\mathrm{a}}^{(0)}\right)\right\}$

where, for some integer $M$ defining the formal order of accuracy,

$$
\begin{aligned}
& \delta_{\mathrm{a}}^{(n)}=f \zeta_{\mathrm{a}}^{(n-1)}-g \nabla^{2} h_{\mathrm{a}}^{(n-1)}-\nabla \cdot(\mathbf{u} \cdot \nabla \mathbf{u})_{\mathrm{a}}^{(n-1)} \\
& (n=1, \ldots, M+1) \\
& \zeta_{\mathrm{a}}^{(n)}=-f \delta_{\mathrm{a}}^{(n-1)}-\nabla \cdot(\mathbf{u} \zeta)_{\mathrm{a}}^{(n-1)}(n=1, \ldots, M) \\
& h_{\mathrm{a}}^{(n)}=-H \delta_{\mathrm{a}}^{(n-1)}-\nabla \cdot(\mathbf{u} h)_{\mathrm{a}}^{(n-1)}(n=1, \ldots, M) \\
& \mathbf{u}_{\mathrm{a}}^{(n)}=\mathbf{u}_{\mathrm{a} \zeta}^{(n)}+\mathbf{u}_{\mathrm{a} \delta}^{(n)} \\
& (n=0, \ldots, M)
\end{aligned}
$$

\footnotetext{
${ }^{3} \mathrm{~A}$ squared norm such as $\epsilon^{2}:=\iint\left(\delta_{\mathrm{a}}^{(0)}-\delta\right)^{2} d x d y$ can be regarded as an autonomous measure of the inaccuracy of the balanced model and, therefore, of the corresponding PV inversion. As in Sect. 7 of McIntyre and Norton (2000), minimization of quantities like $\epsilon$ over reference frames can be used to make the system Galilean invariant; and it is becoming apparent that the favoured reference frames are those in which the jets are most river-like, i.e. in which the meander shapes are closest to stationary.
} 
with $^{4}$

$\mathbf{u}_{\mathrm{a} \zeta}^{(n)}=\hat{\mathbf{z}} \times \nabla \nabla^{-2} \zeta_{\mathrm{a}}^{(n)} \quad$ and $\quad \mathbf{u}_{\mathrm{a} \delta}^{(n)}=\nabla \nabla^{-2} \delta_{\mathrm{a}}^{(n)}$

and

$\delta_{\mathrm{a}}^{(M)}=\delta_{\mathrm{a}}^{(M+1)}=0$.

The notation ()$_{\mathrm{a}}^{(n)}$ outside a product is defined to mean the Leibniz formula for the $n^{\text {th }}$ time derivative of the product with the diagnostic estimates substituted. The last two equations (12) serve to close the system, for given formal accuracy $M$, as counting the variables will verify. Other such closures or truncations are possible. Examples are the truncations first explored in Mohebalhojeh and Dritschel (2001) and summarized in MM07a. The nonlocal functional dependence of $\mathcal{R}$ on the $Q$ field is defined by (9)-(12), for arbitrary $M$, together with (5) and $\zeta_{\mathrm{a}}^{(0)}=\zeta, h_{\mathrm{a}}^{(0)}=h$.

Equations (10a), (10b) and (10c) can be recognized as diagnostic estimates of the exact shallow-water divergence, vorticity and mass-conservation equations and their time derivatives. As already emphasized, the distinction between time derivatives $\partial^{n} / \partial t^{n}$ and their diagnostic estimates is crucial throughout. The only prognostic equation in the system is (4), with its single time derivative. The remaining equations can be regarded as defining an accurate PV inversion operator, delivering the $\mathbf{u}$ and $h$ fields diagnostically when the $Q$ field is given.

This completes the definition of the hyperbalance equations, which in this simplest case are just the equations above, (4)-(12) with $\zeta_{\mathrm{a}}^{(0)}=\zeta$ and $h_{\mathrm{a}}^{(0)}=h$. Numerical integration of this system is of course not trivial. One method is described in MM07b. It was used there in a careful investigation of the actual numerical accuracy, as judged against primitive-equation evolution. The "fuzziness of the slow quasimanifold" (e.g., Warn 1997; Ford et al., 2000 \& refs) precludes convergence to perfect accuracy; and, not surprisingly, the accuracy behaves quasi-asymptotically. It improves at first, as $M$ increases from low values, then deteriorates. At the fairly high Froude and Rossby numbers considered in MM07b, the deterioration begins at $M$ values around 3 or 4 . For lower Froude and Rossby numbers, the quasimanifold is thinner and the deterioration begins at higher $M$ values.

The hyperbalance equations represent what had long seemed to me an unattainable prize, a class of nonHamiltonian PV-conserving balanced models competitive with all known balanced models in terms of accuracy while also, unlike its predecessors, exactly conserving not only mass but also, by implication, all the non-Hamiltonian Casimirs including the potential enstrophy. The competitive accuracy found in MM07b surprised me, and I think would have surprised Rupert also. I for one had long

\footnotetext{
${ }^{4}$ In MM07b there is a typographical error, $(n-1)$ for $(n)$ in Eq. (2.3d), the counterpart of (10d)-(11) above. The error occurs just after the first equals sign.
}

thought that the suppression, in balanced models, of the local mass rearrangement involved in spontaneous-adjustment emission would imply a tradeoff between accuracy and velocity splitting.

It is still possible that MM07b does not represent the last word on this issue. There, the investigation of numerical accuracy was restricted to the shallow-water system. So it remains possible that a tradeoff will be found for two-layer and multi-layer models, in which the path of one jet can cross that of another in a different layer.

The hyperbalance PV inversion operator defined by (5)(12) gives results qualitatively like all the familiar quasigeostrophic results, such as (1), but incomparably more accurate. Like its accurate predecessors it is a weakly nonlinear operator. The corresponding balanced dynamics still involves only a single time derivative acting on a single scalar field - (4) and its generalizations expressing departures from material invariance of PV - with everything else determined diagnostically. So it has the same far-reaching consequences. These include jet self-sharpening and the one-way phase propagation of Rossby waves, quite unlike the two-way propagation of classical waves, for which the time derivatives always occur in pairs.

\section{Concluding remarks: the Taylor identity}

What should be meant by disturbing a jet "in almost any natural way"? The fluid flows under consideration are all characterized by the approximate material invariance of PV, Eq. (4) with an appropriate definition of $Q$. It would seem reasonable, therefore, to class as unnatural any turbulence experiment with artificial forcing that interferes too strongly with PV evolution. Phenomena like PV mixing, jet selfsharpening, and PV staircase formation are unlikely to be well represented in experiments that strongly violate Eq. (4), especially if they violate it in an unnatural or unphysical way. Of course real flows like that on Jupiter, and in the terrestrial atmosphere and oceans, must also violate (4). However, we may safely assume that they violate it not only in a natural way but also much less strongly than in some turbulence experiments.

Thinking about how to disturb a jet naturally is another way to appreciate the basic point that, in the real fluid flows in question, there is no such thing as turbulence without waves. The most obvious natural way to disturb a jet without violating (4) is to send in Rossby waves from somewhere else. If the jet profile is already sharp enough to make it a Rossby waveguide in the horizontal, then for real, baroclinic jets the most natural thing is to send in the waves from above or below. What often happens, in reality, is that the waves propagate or diffract up the jet axis from below, as with ocean jets that feel the bottom topography (Hughes, 1996 \& refs). Other wave sources may serve equally well. One example is low-level baroclinic instability, when nonlinearly saturating 
or occluding (Thorncroft et al., $1993 \&$ refs). In the case of Jupiter, Richard Wood and I are currently exploring the idea that the excitation from below comes directly from large eddies in the thermally-convecting layers beneath the outermost stratified layers, acting somewhat like time-dependent bottom topography.

The requirement for waves to be involved, and therefore radiation stresses, is underlined by the simple fact that, in jet-sharpening or other PV-mixing scenarios, or indeed in almost any thought experiment in which a zonally averaged $\mathrm{PV}$ distribution is changed somehow, PV inversion dictates that the angular momentum distribution must change. It must change in a perfectly definite way, with net change generally nonzero. DM08 gives specific examples. In the simplest PV-mixing scenarios - simple surf-zone or staircasestep formation starting from a uniform background PV gradient - the net angular-momentum change is robustly negative or retrograde, as is easy to show (DM08, Eq. (7.2)). The negative sign is part of the chirality arising from the single time derivative in (4) as with the one-way, retrograde phase propagation of the Rossby waves themselves. Thus jet self-sharpening by Rossby-wave breaking entails decelerating one or both jet flanks, as well as accelerating the core. ${ }^{5}$ And in general there must be a radiation-stress field that mediates the angular-momentum changes. Thus PV mixing is something that in general needs to be "catalysed" by a suitable radiation-stress field, due for instance to momentum transport by Rossby waves, a point further underlined by the well-known Taylor identity, or Taylor-Bretherton identity, Eq. (13) below.

The terrestrial winter stratosphere illustrates all these points in great detail. It efficiently mixes PV over vast areas, mostly in the midlatitude Rossby-wave surf zone. The wellobserved stratospheric global-scale (Brewer-Dobson) circulation is persistently poleward. It is poleward because of the radiation stress. In a zonally averaged description, the winter stratosphere feels a persistent retrograde force arising from the radiation-stress field of the breaking planetaryscale Rossby waves coming up from below, mostly in zonal wavenumbers 1 and 2 . The abovementioned gyroscopicpumping movie shows a case of mostly wave 2 . Not only is the polar night jet being sharpened and re-sharpened throughout the winter - by the efficient PV mixing catalysed by the Rossby-wave radiation or diffraction stress exerted from below - but, on average, around latitude circles intersecting the surf zone, air is being pushed persistently westward. So, in

\footnotetext{
${ }^{5}$ The exceptional case of zero net angular-momentum change is extensively analysed by Dunkerton and Scott (2008). That case can be realized through artificial forcing having zero resultant external force. The simplest such forcing consists of fluctuating force dipoles, producing vortex quadrupoles. It is sometimes discussed under the heading "Welander's massless goldfish" (P. B. Rhines, personal communication). PV pair-production is involved, and therefore PV unmixing as well as mixing.
}

the zonally-averaged dynamics, the strong Coriolis effects try to turn the air poleward. This amounts to a mechanical pumping action, pushing air poleward and then mostly downward (Haynes et al., 1991) against infrared radiative relaxation. It is almost exactly the same thing as Ekman pumping apart from the different origin of the retrograde force. Both things can reasonably be described as cases of "gyroscopic pumping", flagging the crucial role of the strong Coriolis force. There are well known consequences for the distributions of ozone and other greenhouse gases.

To the extent that quasigeostrophic descriptions are qualitatively relevant, and zonally-averaged thought experiments useful, the symbiosis between wavelike and turbulent dynamics is beautifully summarized by the well-known Taylor or Taylor-Bretherton identity (Taylor, 1915; Bretherton, 1966), which is valid for arbitrary disturbance amplitude and therefore applicable to turbulence as well as to waves:

$\overline{v^{\prime} q^{\prime}}=\frac{1}{\rho_{0}}\left(\frac{\partial F}{\partial y}+\frac{\partial G}{\partial z}\right)$.

It says that an eddy PV flux $\overline{v^{\prime} q^{\prime}}$, such as that due to PV mixing, is tied to the radiation-stress divergence. The radiation stress is defined to quasigeostrophic accuracy as having $x y$ and $x z$ components

$(F, G):=\rho_{0}(z)\left(-\overline{u^{\prime} v^{\prime}}, \frac{f_{0} \overline{v^{\prime} \theta^{\prime}}}{N^{2}}\right)$

where $\theta$ is the buoyancy acceleration, $N(z)$ the vertical profile of buoyancy frequency, $f_{0}$ a constant nominal value of the Coriolis parameter, and $\rho_{0}(z)$ a background vertical density profile $\propto \exp \left(-z / H_{p}\right)$ with $H_{p}$ the pressure scale height. The vertical component $G$, crucial to the whole picture sketched above, is what dynamical oceanographers call the form stress across an undulating stratification surface (Bretherton 1969). For historical reasons $(F, G)$ is also called the Eliassen-Palm flux or effective momentum flux. The sign convention in (14) makes flux directions correspond to Rossby-wave group velocities, when applicable. The quasigeostrophic disturbance streamfunction and PV satisfy

$$
\frac{\partial^{2} \psi^{\prime}}{\partial x^{2}}+\frac{\partial^{2} \psi^{\prime}}{\partial y^{2}}+\frac{1}{\rho_{0}} \frac{\partial}{\partial z}\left(\frac{\rho_{0} f_{0}^{2}}{N^{2}} \frac{\partial \psi^{\prime}}{\partial z}\right)=q^{\prime},
$$

from which (13) follows after a line or two of manipulation using $\overline{(}_{x}=0$ and $\left\{u^{\prime}, v^{\prime}, \theta^{\prime}\right\}=\left\{-\psi_{y}^{\prime}, \psi_{x}^{\prime}, f_{0} \psi_{z}^{\prime}\right\}$. The shallow-water counterpart of (13), with $q^{\prime}$ defined as in (2b),

$\overline{v^{\prime} q^{\prime}}=-\frac{\partial}{\partial y} \overline{u^{\prime} v^{\prime}}$

holds regardless of $L_{\mathrm{D}}$ values. So (16) holds even when $L_{\mathrm{D}} \rightarrow \infty$, the case originally considered by Taylor (1915) in another context, here corresponding to an atmosphere with an artificial rigid lid. If steady or time-dependent bottom topography is introduced, then a form-stress term $f_{0} \overline{v^{\prime} \eta^{\prime}}$ 
must be added on the right, where $\eta(x, y, t)$ is topographic height expressed as a fraction of mean layer depth.

Artificial forces do not break (13) and (16). Nor do they break the PV invertibility principle, here expressed by (15) with suitable boundary conditions, unless the artificial forces are so strong as to interfere with balance. It is the rest of the picture that changes. In experiments that strongly violate Eq. (4) the PV flux, $\overline{v^{\prime} q^{\prime}}$, becomes powerless to bring about the kinds of rearrangements of the PV field that we call stirring and mixing. An extreme case would be an artificial forcing, or for that matter an artificial dissipation, that systematically accelerates fluid elements retrogradely or progradely. With such artifices one can generate any jet structure one pleases. The implication is that, in turbulence experiments with artificial forces, it would be useful to pay attention to momentum or impulse injection as well as to energy injection.

Alongside an awareness of the radiation-stress field these considerations impinge on whether we can claim to understand, for instance, the superrotation of equatorial planetary atmospheres such as Jupiter's. Experience with our own terrestrial equatorial superrotation, the stratospheric quasi-biennial oscillation in its prograde or eastward phase (e.g., Baldwin et al., $2001 \&$ refs), calls attention to the radiation-stress fields of several hybrid wave types in addition to those of Rossby and gravity waves. Plumb and Ferrari (2005), Eqs. (22)-(27), go beyond the limitations of quasigeostrophic theory to present some pertinent generalizations of the Taylor identity.

Acknowledgements. I am indebted to Uwe Harlander for his kind encouragement to produce this paper and for invitations to EGU symposia, and to Walter Robinson and the other organizers of the 2006 AGU Chapman Conference on Jets and Annular Structures in Geophysical Fluids, held in January 2006 at Savannah, Georgia. The resulting conversations and emails taught me much about the current state of knowledge in the atmosphere-ocean dynamics and geophysical turbulence communities. Shafer Smith, Andy Thompson, Richard Wood and two anonymous referees helped me to clarify the manuscript. Over a longer timespan John Allen, David Dritschel, Boris Galperin, Peter Haynes, Ali Mohebalhojeh, Warwick Norton, Tim Palmer, Peter Rhines, Ian Roulstone, Ted Shepherd, and the late Peter Killworth - another brilliant, generous, and much-loved colleague - all made important contributions to my understanding of these topics, as did Rupert Ford, who took such joy in what Richard Feynman called the pleasure of finding things out, and who inspired me and so many of my colleagues during his brief but brilliant career. Colleagues may like to be reminded that the UK Royal Meteorological Society administers a Rupert Ford Memorial Fund to assist young scientists.

Edited by: U. Harlander

Reviewed by: two anonymous referees

\section{References}

Armi, L. and Flament, P.: Cautionary remarks on the spectral interpretation of turbulent flows, J. Geophys. Res., 90(C6), 11779$11782,1985$.

Baldwin, M. P., Gray, L. J., Dunkerton, T. J., Hamilton, K., Haynes, P. H., Randel, W. J., Holton, J. R., Alexander, M. J., Hirota, I., Horinouchi, T., Jones, D. B. A., Kinnersley, J. S., Marquardt, C., Sato, K., and Takahashi, M.: The quasi-biennial oscillation, Revs. Geophys., 39, 179-229, 2001.

Baumert, H. Z., Simpson, J. H, and Sündermann, J. (Eds.): Marine Turbulence: Theories, Observations, and Models, Cambridge, University Press, 2005.

Beron-Vera, F. J., Brown, M. G., Olascoaga, M. J., Rypina, I. I., Koçak, H., and Udovydchenkov, I. A.: Zonal jets as transport barriers in planetary atmospheres, J. Atmos. Sci., in press, 2008.

Bretherton, F. P.: Critical layer instability in baroclinic flows. Q. J. Roy. Meteorol. Soc., 92, 325-334, 1966.

Bretherton, F. P.: Momentum transport by gravity waves, Q. J. Roy. Meteorol. Soc., 95, 213-243, 1969.

Cho, Y.-K. and Polvani, L. M.: The emergence of jets and vortices in freely-evolving shallow-water turbulence on a sphere, Phys. Fluids, 8, 1531-1540, 1996.

Danilov, S. and Gryanik, V. M.: Barotropic beta-plane turbulence in a regime with strong zonal jets revisited, J. Atmos. Sci., 61, 2283-2295, 2004.

Danilov, S. and Gurarie, D.: Scaling, spectra and zonal jets in betaplane turbulence, Phys. Fluids, 16, 2592-2603, 2004.

Dickinson, R. E.: Theory of planetary wave-zonal flow interaction, J. Atmos. Sci., 26, 73-81, 1969.

Dritschel, D. G. and McIntyre, M. E.: Multiple jets as PV staircases: the Phillips effect and the resilience of eddy-transport barriers, J. Atmos. Sci., 65, 855-874, 2008.

Dunkerton, T. J. and Scott, R. K.: A barotropic model of the angular momentum conserving potential vorticity staircase in spherical geometry, J. Atmos. Sci., 65, 1105-1136, 2008.

Esler, J. G.: The turbulent equilibration of an unstable baroclinic jet, J. Fluid Mech., 599, 241-268, 2008.

Ford, R., McIntyre, M. E., and Norton, W. A.: Balance and the slow quasimanifold: some explicit results, J. Atmos. Sci., 57, 1236$1254,2000$.

Ford, R., McIntyre, M. E., and Norton, W. A.: Reply to Comments by S. Saujani and T. G. Shepherd on "Balance and the slow quasimanifold: some explicit results", J. Atmos. Sci., 59, 2878-2882, 2002.

Gleick, J.: Genius: Richard Feynman and Modern Physics, Little Brown, London, 1992.

Haynes, P. H.: The effect of barotropic instability on the nonlinear evolution of a Rossby-wave critical layer, J. Fluid Mech., 207, 231-266, 1989.

Haynes, P. H., Marks, C. J., McIntyre, M. E., Shepherd, T. G., and Shine, K. P.: On the "downward control" of extratropical diabatic circulations by eddy-induced mean zonal forces, J. Atmos. Sci., 48, 651-678, 1991. Also J. Atmos. Sci., 53, 2105-2107, 1996.

Haynes, P. H., Poet, D. A., and Shuckburgh, E. F.: Transport and mixing in kinematic and dynamically-consistent flows, J. Atmos. Sci., 64, 3640-3651, 2007.

Held, I. M. and Phillips, P. J.: Linear and nonlinear barotropic decay on the sphere, J. Atmos. Sci., 44, 200-207, 1987. 
Hoskins, B. J., McIntyre, M. E., and Robertson, A. W.: On the use and significance of isentropic potential-vorticity maps, Q. J. Roy. Meteor. Soc., 111, 877-946, 1985. Corrigendum 113, 402-404, 1987.

Hughes, C. W.: The Antarctic Circumpolar Current as a waveguide for Rossby waves, J. Phys. Oceanogr., 26, 1375-1387, 1996.

Humphreys, T. and Marcus, P. S.: Vortex street dynamics: the selection mechanism for the areas and locations of Jupiter's vortices. J. Atmos. Sci., 64, 1318-1333, 2007.

Juckes, M. N. and McIntyre, M. E.: A high resolution, one-layer model of breaking planetary waves in the stratosphere, Nature, 328, 590-596, 1987.

Killworth, P. D. and McIntyre, M. E.: Do Rossby-wave critical layers absorb, reflect or over-reflect? J. Fluid Mech., 161, 449-492, 1985.

Legras, B., Dritschel, D. G., and Caillol, P.: The erosion of a distributed two-dimensional vortex in a background straining flow, J. Fluid Mech., 441, 369-398, 2001.

Marcus, P. S.: Jupiter's great red spot and other vortices, Ann. Rev. Astron. Astrophys., 31, 523-573, 1993.

Marcus, P. S. and Lee, C.: A model for eastward and westward jets in laboratory experiments and planetary atmospheres Phys. Fluids, 10, 1474-1489, 1998.

McIntyre, M. E.: Obituary: Rupert Ford, Q. J. Roy. Meteor. Soc., 127, 1489-1490, 2001.

McIntyre, M. E. and Norton, W. A.: Dissipative wave-mean interactions and the transport of vorticity or potential vorticity, J. Fluid Mech., 212, 403-435, 1990. Corrigendum 220, 693, 1990.

McIntyre, M. E. and Norton, W. A.: Potential vorticity inversion on a hemisphere, J. Atmos. Sci., 57, 1214-1235, 2000. Corrigendum, 58, 949, 2001.

McIntyre, M. E. and Palmer, T. N.: A note on the general concept of wave breaking for Rossby and gravity waves, Pure Appl. Geophys., 123, 964-975, 1985.

McIntyre, M. E. and Roulstone, I.: Are there higher-accuracy analogues of semigeostrophic theory?, in: Large-scale AtmosphereOcean Dynamics, Vol. II: Geometric Methods and Models, edited by: Norbury, J. and Roulstone, I., Cambridge, University Press, 301-364, 2002.

Mohebalhojeh, A. R.: On shallow-water potential-vorticity inversion by Rossby number expansions, Q. J. Roy. Meteor. Soc., 128, 679-694, 2002.

Mohebalhojeh, A. R. and Dritschel, D. G.: Hierarchies of balance conditions for the $f$-plane shallow water equations, J. Atmos. Sci., 58, 2411-2426, 2001.

Mohebalhojeh, A. R. and McIntyre, M. E.: Local mass conservation and velocity splitting in PV-based balanced models. Part I: The hyperbalance equations, J. Atmos. Sci., 64, 1782-1793, 2007a.

Mohebalhojeh, A. R. and McIntyre, M. E.: Local mass conservation and velocity splitting in PV-based balanced models. Part II: Numerical results, J. Atmos. Sci., 64, 1794-1810, 2007b.

Niiler, P. P., Maximenko, N. A., Panteleev, G. G., Yamagata, T., and Olson, D. B.: Near-surface dynamical structure of the Kuroshio Extension, J. Geophys. Res., 108(C6), 3193, doi:10.1029/2002JC001461, 2003.
Norton, W. A.: Balance and potential vorticity inversion in atmospheric dynamics, University of Cambridge, $\mathrm{PhD}$ Thesis (available from Cambridge University Library, Superintendent of Manuscripts), 167 pp., 1988.

Nycander, J., Dritschel, D. G., and Sutyrin, G. G.: The dynamics of long frontal waves in the shallow water equations, Phys. Fluids, 5(5), 1089-1091, 1993.

Peltier, W. R. and Stuhne, G. R.: The upscale turbulent cascade: shear layers, cyclones and gas giant bands, in: Meteorology at the Millennium, edited by: Pearce, R. P., London, Academic, 43-61, 2002.

Phillips, O. M.: Turbulence in a strongly stratified fluid - is it unstable? Deep Sea Res., 19, 79-81, 1972.

Plumb, R. A. and Ferrari, R.: Transformed Eulerian-mean theory. Part I: Nongeostrophic theory for eddies on a zonal-mean flow, J. Phys. Oceanogr., 35, 165-174, 2005.

Polvani, L. M. and Plumb, R. A.: Rossby wave breaking, microbreaking, filamentation and secondary vortex formation: the dynamics of a perturbed vortex, J. Atmos. Sci., 49, 462-476, 1992.

Read, P. L., Yamazaki, Y. H., Lewis, S. R., Williams, P. D., Wordsworth, R., Miki-Yamazaki, K., Sommeria, J., and Didelle, H.: Dynamics of convectively driven banded jets in the laboratory, J. Atmos. Sci., 64, 4031-4052, 2007.

Riese, M., Manney, G. L., Oberheide, J., Tie, X., Spang, R., and Küll, V.: Stratospheric transport by planetary wave mixing as observed during CRISTA-2, J. Geophys. Res., 107(D23), 8179, doi:10.1029/2001JD000629, 2002.

Rossby, C.-G.: Dynamics of steady ocean currents in the light of experimental fluid mechanics, Mass. Inst. of Technol. \& Woods Hole Oc. Instn., Phys. Oc. Meteorol., 5(1), 1-43, 1936.

Rypina, I. I., Brown, M. G., Beron-Vera, F. J., Olascoaga, M. J., Koçak, H., and Udovydchenkov, I. A.: On the Lagrangian dynamics of atmospheric zonal jets and the permeability of the stratospheric polar vortex, J. Atmos. Sci., 64, 3595-3610, 2007.

Scott, R. K. and Polvani, L. M.: Forced dissipative shallow water flow on the sphere: equatorial confinement of zonal jets, J. Atmos. Sci., 64, 3158-3176, 2007.

Simmons, A. J.: Planetary-scale disturbances in the polar winter stratosphere, Q. J. Roy. Meteor. Soc., 100, 76-108, 1974.

Sommeria, J., Meyers, S. D., and Swinney, H. L.: Laboratory model of a planetary eastward jet, Nature, 337, 58-61, 1989.

Sommeria, J., Meyers, S. D., and Swinney, H. L.: Experiments on vortices and Rossby waves in eastward and westward jets, in: Nonlinear Topics in Ocean Physics, edited by: Osborne,A. R., Amsterdam, North-Holland, 227-269, 1991.

Stewartson, K.: The evolution of the critical layer of a Rossby wave, Geophys. Astrophys. Fluid Dyn., 9, 185-200, 1978.

Taylor, G. I.: Eddy motion in the atmosphere, Phil. Trans. Roy. Soc. Lond., A215, 1-23, 1915.

Thorncroft, C. D., Hoskins, B. J., and McIntyre, M. E.: Two paradigms of baroclinic-wave life-cycle behaviour, Q. J. Roy. Meteor. Soc., 119, 17-55, 1993.

Warn, T. and Warn, H.: The evolution of a nonlinear critical level, Stud. Appl. Math., 59, 37-71 1978.

Warn, T.: Nonlinear balance and quasi-geostrophic sets, Atmos.Ocean, 35, 135-145, 1997. 